\title{
Short communication: Detection and molecular characterization of methicillin-resistant Staphylococcus aureus isolated from subclinical bovine mastitis cases in China
}

\author{
Feng Yang, ${ }^{*}$ ㄴ) Shidong Zhang, $\odot$ Xiaofei Shang, Hongsheng Li, Hang Zhang, $\odot$ Dongan Cui, Xurong Wang, \\ Ling Wang, Zuoting Yan, and Yan Sun* \\ Engineering and Technology Research Center of Traditional Chinese Veterinary Medicine of Gansu Province, \\ Key Laboratory of New Animal Drug Project of Gansu Province, Key Laboratory of Veterinary Pharmaceutical Development \\ of Ministry of Agriculture, Lanzhou Institute of Husbandry and Pharmaceutical Sciences of Chinese Academy of Agricultural Science, \\ Lanzhou 730050, PR China
}

\section{ABSTRACT}

This study investigated the antimicrobial susceptibility of methicillin-resistant Staphylococcus aureus (MRSA) isolated from cases of subclinical bovine mastitis in China, as well as resistance mechanisms and virulence genes encoding adhesins and toxins. We determined antimicrobial susceptibility using the disk diffusion method, and analyzed resistance, adhesin, and toxin genes using PCR. We confirmed MRSA in 73 of 498 (14.7\%) Staph. aureus isolates recovered from subclinical mastitic milk samples. All isolates were positive for mecA. The MRSA isolates showed high resistance to penicillin (100.0\%), gentamicin (100.0\%), and tetracycline (98.6\%). All MRSA isolates harbored resistance genes blaZ (penicillin), aacA/aphD (gentamicin), and tet $M$ (alone or in combination with tetK, tetracycline). Moreover, all isolates carried the adhesin genes fnbp $A$, clfA, clfB, cna, sdrE, and map/eap, and most carried $s d r C(98.6 \%), \operatorname{sdrD}(95.9 \%), b b p(94.5 \%)$, and $e b p S$ (80.8\%). The toxin genes seh, hla, and hld were present in all isolates, and most isolates carried sea $(71.2 \%)$, seg $(84.9 \%)$, sei $(82.2 \%)$, lukE-lukD $(97.3 \%)$, and hlg $(72.6 \%)$. These findings of high-level resistance to antimicrobials commonly used in dairy cattle should lead to calls for antibiogram analysis before antimicrobial therapy. The high frequency of adhesin and toxin genes in MRSA indicates their potential virulence in bovine mastitis in China.

Key words: methicillin-resistant Staphylococcus aureus, bovine mastitis, antimicrobial resistance, virulence gene

Received January 17, 2019.

Accepted August 30, 2019.

*Corresponding authors: yangfeng@caas.cn and 516845664@qq.com

\section{Short Communication}

Bovine mastitis is a highly prevalent and costly disease for the dairy industry worldwide (Yang et al., 2018). Staphylococcus aureus is a major etiological pathogen that causes bovine mastitis. Antimicrobial therapy is a primary tool for controlling staphylococcal mastitis (Wang et al., 2018), but the cure rate is low because of increasing antimicrobial resistance developed by this pathogen, especially methicillin-resistant Staph. aureus (MRSA). Indeed, MRSA has shown resistance to all types of $\beta$-lactam antibiotics currently available for mastitis therapy (Vanderhaeghen et al., 2010).

Methicillin-resistant Staph. aureus are considered a serious threat to public health because they can be easily spread through healthy carriers, and they express a large number of virulence factors, including toxins, cell wall-associated adhesins, and secreted exoproteins, making infection more likely (Herrera et al., 2016). Recently, the number of publications describing the detection of MRSA in milk or dairy products has grown rapidly worldwide (Holmes and Zadoks, 2011; Seixas et al., 2014; Al-Ashmawy et al., 2016; Guimarães et al., 2017; Klibi et al., 2018). However, little is known about the virulence gene profiles of MRSA from bovine mastitis; in China, only a few reports exist of some virulence determinants of limited MRSA isolates $(\mathrm{Pu}$ et al., 2014; Li et al., 2017b; Wang et al., 2018). We designed the current study to investigate the antimicrobial susceptibility of, resistance mechanisms implicated in, and virulence genes encoding adhesins and toxins of MRSA isolates from cases of subclinical bovine mastitis in China.

The MRSA strains investigated in this study were confirmed from 498 Staph. aureus isolates recovered from 3,136 cases of subclinical bovine mastitis. The mastitic milk samples were obtained from 102 commercial dairy herds in 19 provinces in China from August 
2013 to April 2018, as described in our previous study (Yang et al., 2018). We identified Staph. aureus by morphological characterization and biochemical testing, as previously described (Cressier and Bissonnette, 2011). The MRSA strains were confirmed by the cefoxitin $(30 \mu \mathrm{g})$ disk diffusion method according to the recommendations of the Clinical and Laboratory Standards Institute (CLSI; CLSI, 2013).

We performed antimicrobial susceptibility testing using the disk diffusion method on Mueller-Hinton agar (Oxoid, Basingstoke, UK) according to CLSI criteria (CLSI, 2013). We applied the following panel of antimicrobial agents (Oxoid): penicillin (10 units), gentamicin $(10 \mu \mathrm{g})$, tetracycline $(30 \mu \mathrm{g})$, nitrofurantoin $(300 \mu \mathrm{g})$, trimethoprim/sulfamethoxazole $(1.25 / 23.75 \mu \mathrm{g})$, chloramphenicol $(30 \mu \mathrm{g})$, quinupristin/dalfopristin (15 $\mu \mathrm{g})$, and linezolid $(30 \mu \mathrm{g})$. We used Staph. aureus ATCC 25923 as a positive control strain.

We extracted bacterial genomic DNA using the Bacterial DNA Kit (Omega Bio-Tek, Norcross, GA; Yang et al., 2018). The corresponding resistance genes for methicillin (mecA and mecC), penicillin (blaZ), gentamicin $(a a c A / a p h D)$, and tetracycline (tetK and tet $M$ ) were searched using PCR as previously described (Paterson et al., 2012; Yang et al., 2016). We also performed PCR assays as described previously for adhesin genes encoding fibronectin bind protein (FnBPA; fnbpA), clumping factor (ClfA and ClfB; clfA and clfB), collagen binding protein ( $\mathrm{Cna}$; cna), serine-aspartic acid repeat proteins ( $\mathrm{SdrC}, \mathrm{SdrD}$, and $\mathrm{SdrE}$; $s d r C$, sdrD, and $s d r E$ ), bone sialoprotein-binding protein (Bbp; $b b p)$, elastin-binding protein (EbpS; ebpS), and major histocompatibility complex class II analogous protein/ extracellular adherence protein (Map/Eap; map/eap; Peacock et al., 2002). In addition, we evaluated the toxin genes encoding staphylococcal enterotoxins (sea, seb, sec, sed, see, seg, seh, sei, sej, sen, seo, and sem), toxic shock syndrome toxin-1 (TSST-1; tst), exfoliative toxins (eta and etb), leukocidins (PVL, LukE-LukD, and LukM; lukPV, lukE-lukD, and lukM), hemolysins ( $\alpha$-hemolysin, $\beta$-hemolysin, $\gamma$-hemolysin, $\delta$-hemolysin; $h l a, h l b, h l d$, and $h l g$ ), and epidermal cell differentiation inhibitor (EDIN; edin) using PCR and following a method described previously (Jarraud et al., 2002). The PCR products were analyzed using $1.2 \%$ agarose gel electrophoresis.

Among 498 Staph. aureus isolates, we identified 73 $(14.7 \%)$ as MRSA based on the cefoxitin disk diffusion method. The antimicrobial resistance profiles of the MRSA isolates are listed in Table 1. All MRSA isolates were resistant to penicillin and gentamicin, but susceptible to nitrofurantoin, trimethoprim/sulfamethoxazole, quinupristin/dalfopristin, and linezolid.
Resistance to tetracycline was detected at high rate in $72(98.6 \%)$ isolates. Only 2 isolates were resistant to chloramphenicol $(2.7 \%)$.

All MRSA isolates carried $>4$ of the tested resistance genes (Table 1). The resistance genes mecA, blaZ, and aacA/aphD were present in all isolates, but mec $C$ was not found in any isolates. All isolates harbored the tetracycline resistance gene tet $M(100.0 \%)$, either alone or in combination with tetK $(95.9 \%)$, including 1 (1.4\%) tetracycline-susceptible isolate.

The presence and distribution of adhesin and toxin genes in the MRSA isolates are listed in Table 1. All MRSA isolates harbored the adhesin genes fnbp A, clfA, clfB, cna, sdrE, and map/eap, and most contained $s d r C(98.6 \%, 72 / 73), \operatorname{sdrD}(95.9 \%, 70 / 73), b b p(94.5 \%$, $69 / 73)$, and $\operatorname{ebpS}(80.8 \%, 59 / 73)$. All tested isolates contained the toxin genes seh, hla, and hld, and most contained sea $(71.2 \%, 52 / 73)$, seg $(84.9 \%, 62 / 73)$, sei $(82.2 \%, 60 / 73)$, lukE-lukD $(97.3 \%, 71 / 73)$, and $h l g$ $(72.6 \%, 53 / 73)$. Fewer isolates contained seb $(19.2 \%$, $14 / 73)$, sec $(19.2 \%, 14 / 73)$, sen $(30.1 \%, 22 / 73)$, seo (30.1\%, 22/73), sem $(28.8 \%, 21 / 73)$, tst $(28.8 \%, 21 / 73)$, lukPV $(2.7 \%, 2 / 73)$, and $h l b(23.3 \%, 17 / 73)$. None of the 73 MRSA isolates carried the toxin genes sed, see, sej, eta, etb, or edin.

Although the prevalence of MRSA in bovine mastitis is generally low (Bardiau et al., 2013; Seixas et al., 2014), the emergence and spread of MRSA is becoming a major animal and public health problem worldwide (Parisi et al., 2016; Klibi et al., 2018). Previous studies have shown that the occurrence of MRSA from bovine mastitis varied from 1.4 to $47.6 \%$ in China ( $\mathrm{Pu}$ et al., 2014; Wang et al., 2018). In the present study, $14.7 \%$ of Staph. aureus isolates were identified as MRSA. Our findings were in line with studies conducted in other countries, which revealed that the prevalence of MRSA was $9.3 \%$ in Belgium, $17.2 \%$ in Turkey, $13.9 \%$ in Korea, and $6.6 \%$ in Tunisia (Türkyilmaz et al., 2010; Vanderhaeghen et al., 2010; Song et al., 2016; Klibi et al., 2018). In contrast, MRSA has been detected in bovine mastitis more frequently in India (48.7\%; Mistry et al., 2016). These variations in frequencies may be due to different sample sizes, different seasons, and geographical discrepancies (Klibi et al., 2018).

In the current study, all MRSA isolates harbored the methicillin-resistant gene mecA, but no isolate carried mec $C$. Some mec $C$-positive MRSA isolates from bovine milk have been reported in different countries (Prenafeta et al., 2014; Herrera et al., 2016). However, to the best of our knowledge, mec $C$-positive MRSA from bovine mastitis in China has not been detected to date. In the current study, all MRSA isolates showed resistance to penicillin and gentamicin, and most displayed 
Yang et al.: SHORT COMMUNICATION: ANTIMICROBIAL RESISTANCE AND VIRULENCE OF MRSA

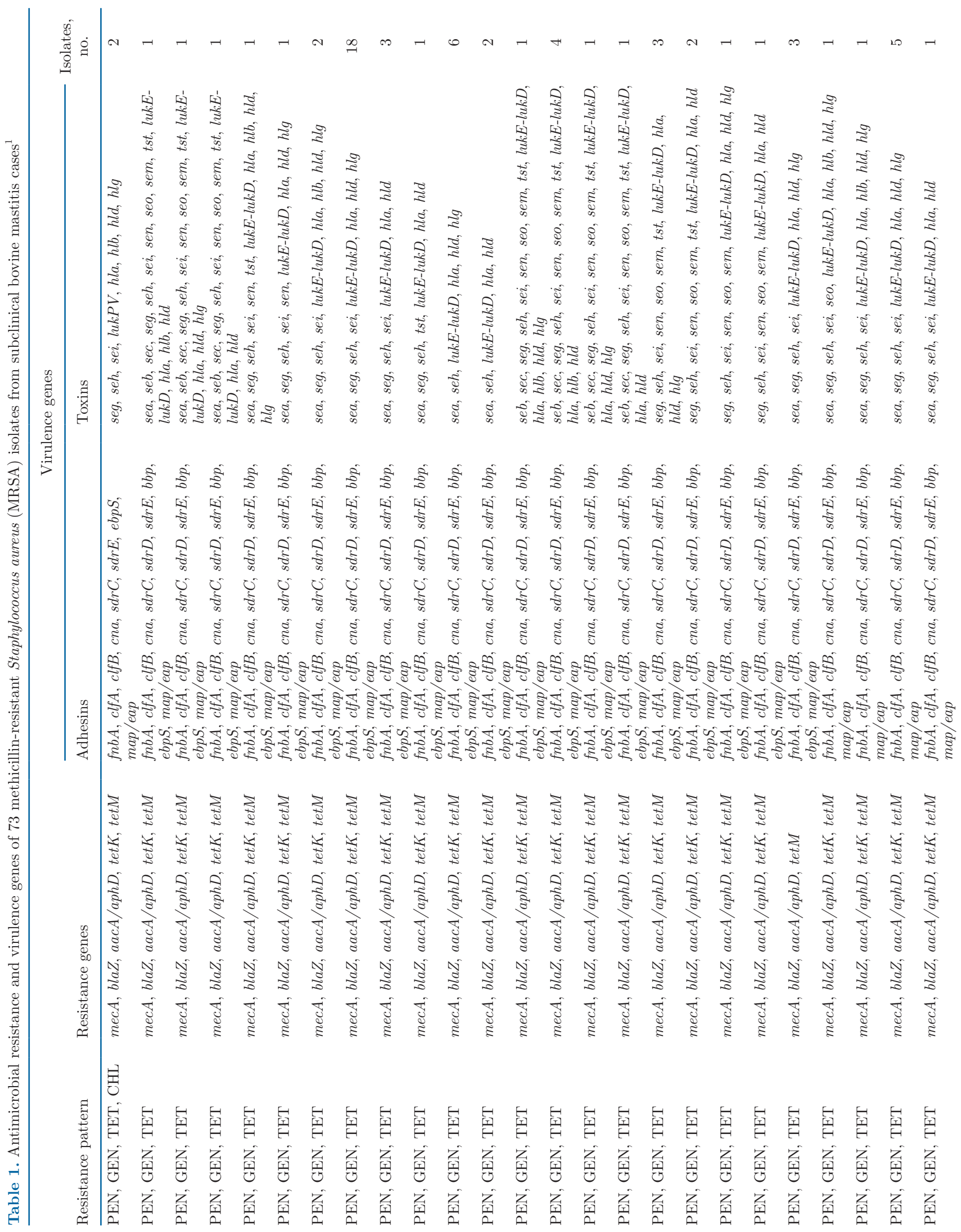

Journal of Dairy Science Vol. 103 No. 1, 2020 
Yang et al.: SHORT COMMUNICATION: ANTIMICROBIAL RESISTANCE AND VIRULENCE OF MRSA

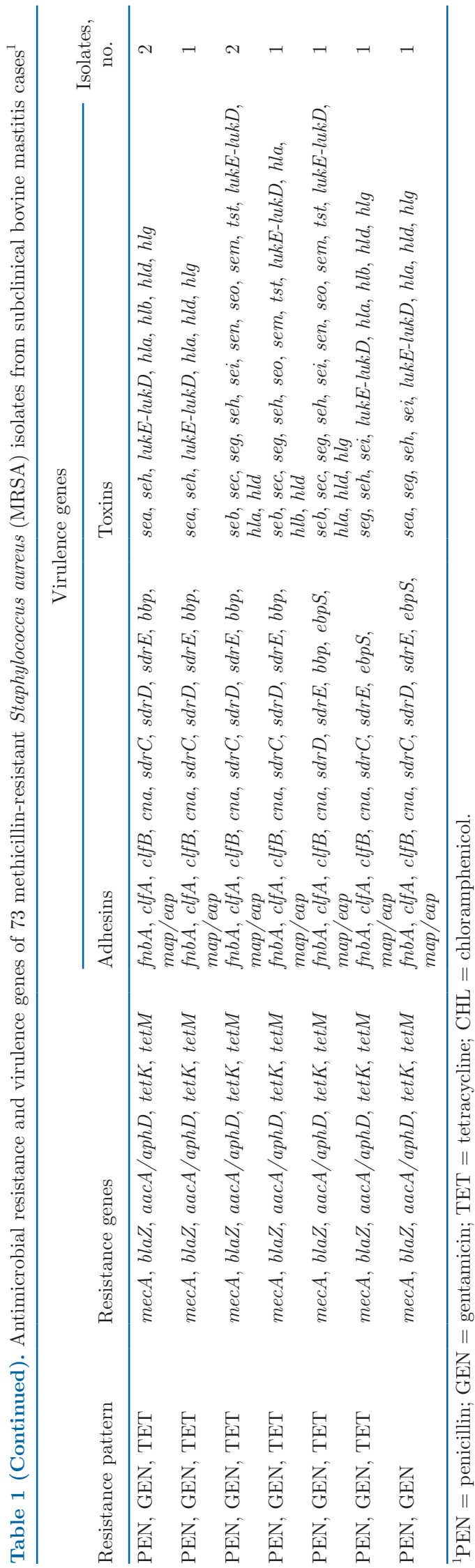

resistance to tetracycline. All of these isolates harbored the resistance-related genes blaZ (penicillin), aacA/ $a p h D$ (gentamicin), and tet $M$ (tetracycline, alone or in combination with tetK). Similar high-level phenotypic and genotypic resistance to these antimicrobial agents has been frequently observed in MRSA from bovine mastitis in other studies (Türkyilmaz et al., 2010; Bardiau et al., 2013; Silva et al., 2014). This high resistance rate and the presence of resistance-related genes could be explained by the frequent use of these antimicrobials in dairy cattle in China ( $\mathrm{Li}$ et al., 2017a; Liu et al., 2017). Interestingly, 1 tetracycline-susceptible MRSA strain carried both tet $K$ and tet $M$ genes. This discrepancy may have been due to the lack of expression of the resistance genes (Hammad et al., 2014).

Staphylococcus aureus can express various adhesins that are essential for bacterial colonization to host cells (McCarthy and Lindsay, 2010). Fibronectin bind protein $(f n b p A)$, clumping factors (clfA and $c l f B)$, and collagen binding protein ( $c n a$ ) are important adhesins for binding to host cells, colonization, and invasion (Aslantaş and Demir, 2016). In the current study, all MRSA isolates harbored the adhesin genes fnbp A, clfA, $c l f B$, and $c n a$, similar to previous studies in China and other countries (Aslantaş and Demir, 2016; Locatelli et al., 2017; Wang et al., 2018). Putative cell-surface proteins encoded by $s d r C, s d r D$, and $s d r E$ could recognize adhesive matrix moleculars and promote Staph. aureus adhesion to host cells (Tung et al., 2000; Corrigan et al., 2009; Yu et al., 2012). In the current study, we found that all MRSA carried $s d r E$, and most of them contained $s d r C$ and $s d r D$, in agreement with previous studies (Yu et al., 2012; Cheraghi et al., 2017). In addition, we detected the $b b p$ and $e b p S$ genes in 94.5 and $80.8 \%$ of the MRSA isolates, respectively; Bbp and EbpS can mediate the binding of Staph. aureus to surface proteins or soluble elastin peptides in host cells (Aung et al., 2011). Our findings agreed with those reported by others with respect to the presence of $b b p$ and $e b p S$ (Aung et al., 2011; Tang et al., 2013). Moreover, all MRSA isolates in the current study carried map/eap, in agreement with previous human studies (Peacock et al., 2002; Cheraghi et al., 2017). Adhesive proteins Map and Eap have been associated with the adhesive interactions of Staph. aureus with a broad spectrum of host molecules (Hussain et al., 2002). To the best of our knowledge, this is the first report of map/eap in MRSA recovered from bovine mastitis in China.

In addition to adhesins, Staph. aureus can produce a wide range of toxins involved in the invasion of host cells and the evasion of immune response (Seixas et al., 2014). We determined the presence of toxin genes among the 73 MRSA isolates in the current study. It has been suggested that toxins SEs and TSST-1 may 
play a role in the development of bovine mastitis by providing an attractive environment for staphylococcocal colonization (Artursson et al., 2016). In the current study, all MRSA isolates harbored at least 2 staphylococcal enterotoxin genes; seh, seg, sei, and sea were the most frequently detected. These findings were in accordance with those of other reports (Yu et al., 2012; Song et al., 2016; Klibi et al., 2018; Wang et al., 2018). Moreover, $28.8 \%$ of the isolates contained tst in this study, in line with previous studies in China (Wang et al., 2016; Zhang et al., 2018). Leukotoxins and hemolysins are thought to play important roles in the initiation or exacerbation of mastitis. They are 2-component toxins that kill erythrocytes, phagocytes, or both by forming pores in host cell membranes (Fueyo et al., 2005; Chalmeau et al., 2011). Similar to previous studies (Fueyo et al., 2005; Al-Ashmawy et al., 2016; Wang et al., 2018), we found that most of the MRSA contained lukE-lukD (97.3\%). We observed lukPV in only $2.7 \%$ of isolates, although it has been reported that $\operatorname{lukPV}$ commonly presents at a high prevalence among methicillin-sensitive isolates (Wang et al., 2018). Furthermore, all MRSA isolates carried hla and hld, and many carried $h l g(72.6 \%)$ and $h l b(23.3 \%)$. These findings were in agreement with other recent studies, which reported that $h l a$ and $h l d$ genes were frequently observed in Staph. aureus (Xu et al., 2015; Wang et al., 2016).

In conclusion, although the prevalence of MRSA was low, the isolates in the current study exhibited a high rate of phenotypic and genotypic resistance to antimicrobials commonly used in dairy cattle in China. The potential risk of MRSA transmission via the food chain cannot be ignored. In addition, the high occurrence of all adhesin genes tested in this study and the toxin genes sea, seg, seh, sei, lukE-lukD, hla, hld, and hlg in MRSA indicate their pathogetic potential in bovine mastitis in China. However, bovine mastitis caused by MRSA cannot be explained by the action of an individual virulence factor (Peacock et al., 2002). Further studies need to be conducted to explore the combined action of these factors in MRSA pathogenesis.

\section{ACKNOWLEDGMENTS}

This work was supported by the National Key Research and Development Program of China (grant no. 2017YFD0502200), Lanzhou; the National Natural Science Foundation of China (grant no. 31802232), Lanzhou; the Supervision of Quality and Safety of Agricultural Products (grant no. 2130109) Lanzhou; and the Key Research and Development Program of Ningxia (grant no. 2019BEF02003), Ningxia.

\section{REFERENCES}

Al-Ashmawy, M. A., K. I. Sallam, S. M. Abd-Elghany, M. Elhadidy, and T. Tamura. 2016. Prevalence, molecular characterization, and antimicrobial susceptibility of methicillin-resistant Staphylococcus aureus isolated from milk and dairy products. Foodborne Pathog. Dis. $13: 156-162$.

Artursson, K., R. Söderlund, L. Liu, S. Monecke, and J. Schelin. 2016. Genotyping of Staphylococcus aureus in bovine mastitis and correlation to phenotypic characteristics. Vet. Microbiol. 193:156-161.

Aslantaş, Ö., and C. Demir. 2016. Investigation of the antibiotic resistance and biofilm-forming ability of Staphylococcus aureus from subclinical bovine mastitis cases. J. Dairy Sci. 99:8607-8613.

Aung, M. S., N. Urushibara, M. Kawaguchiya, T. S. Aung, S. Mya, T. San, K. M. Nwe, and N. Kobayashi. 2011. Virulence factors and genetic characteristics of methicillin-resistant and -susceptible Staphylococcus aureus isolates in Myanmar. Microb. Drug Resist. $17: 525-535$

Bardiau, M., K. Yamazaki, J. N. Duprez, B. Taminiau, J. G. Mainil, and I. Ote. 2013. Genotypic and phenotypic characterization of methicillin-resistant Staphylococcus aureus (MRSA) isolated from milk of bovine mastitis. Lett. Appl. Microbiol. 57:181-186.

Chalmeau, J., N. Monina, J. Shin, C. Vieu, and V. Noireaux. 2011. $\alpha$-Hemolysin pore formation into a supported phospholipid bilayer using cell-free expression. Biochim. Biophys. Acta 1808:271-278.

Cheraghi, S., L. Pourgholi, M. Shafaati, S. H. Fesharaki, A. Jalali, R. Nosrati, and M. A. Boroumand. 2017. Analysis of virulence genes and accessory gene regulator $(a g r)$ types among methicillin-resistant Staphylococcus aureus strains in Iran. J. Glob. Antimicrob. Resist. 10:315-320.

CLSI (Clinical and Laboratory Standards Institute). 2013. Performance Standards for Antimicrobial Susceptibility Testing. CLSI document M100-S23. Clinical and Laboratory Standards Institute, Wayne, PA.

Corrigan, R. M., H. Miajlovic, and T. J. Foster. 2009. Surface proteins that promote adherence of Staphylococcus aureus to human desquamated nasal epithelial cells. BMC Microbiol. 9:22.

Cressier, B., and N. Bissonnette. 2011. Assessment of an extraction protocol to detect the major mastitis-causing pathogens in bovine milk. J. Dairy Sci. 94:2171-2184.

Fueyo, J. M., M. C. Mendoza, M. R. Rodicio, J. Muñiz, M. A. Alvarez, and M. C. Martín. 2005. Cytotoxin and pyrogenic toxin superantigen gene profiles of Staphylococcus aureus associated with subclinical mastitis in dairy cows and relationships with macrorestriction genomic profiles. J. Clin. Microbiol. 43:1278-1284.

Guimarães, F. F., M. P. Manzi, S. F. Joaquim, V. B. Richini-Pereira, and H. Langoni. 2017. Short communication: Outbreak of methicillin-resistant Staphylococcus aureus (MRSA)-associated mastitis in a closed dairy herd. J. Dairy Sci. 100:726-730.

Hammad, A. M., T. Shimamoto, and T. Shimamoto. 2014. Genetic characterization of antibiotic resistance and virulence factors in Enterococcus spp. from Japanese retail ready-to-eat raw fish. Food Microbiol. 38:62-66.

Herrera, F. C., M. L. García-López, and J. A. Santos. 2016. Short communication: Characterization of methicillin-resistant Staphylococcus aureus isolated from raw milk fresh cheese in Colombia. J. Dairy Sci. 99:7872-7876.

Holmes, M. A., and R. N. Zadoks. 2011. Methicillin resistant S. aureus in human and bovine mastitis. J. Mammary Gland Biol. Neoplasia $16: 373-382$.

Hussain, M., A. Haggar, C. Heilmann, G. Peters, J. I. Flock, and M. Herrmann. 2002. Insertional inactivation of Eap in Staphylococcus aureus strain Newman confers reduced staphylococcal binding to fibroblasts. Infect. Immun. 70:2933-2940.

Jarraud, S., C. Mougel, J. Thioulouse, G. Lina, H. Meugnier, F. Forey, X. Nesme, J. Etienne, and F. Vandenesch. 2002. Relationships between Staphylococcus aureus genetic background, virulence factors, agr groups (alleles), and human disease. Infect. Immun. 70:631-641.

Klibi, A., A. Jouini, P. Gómez, K. Slimene, S. Ceballos, C. Torres, and A. Maaroufi. 2018. Molecular characterization and clonal diversity 
of methicillin-resistant and -susceptible Staphylococcus aureus isolates of milk of cows with clinical mastitis in Tunisia. Microb. Drug Resist. 24:1210-1216.

Li, J., N. Jiang, Y. Ke, A. T. Feßler, Y. Wang, S. Schwarz, and C. Wu. 2017a. Characterization of pig-associated methicillin-resistant Staphylococcus aureus. Vet. Microbiol. 201:183-187.

Li, T., H. Lu, X. Wang, Q. Gao, Y. Dai, J. Shang, and M. Li. 2017b. Molecular characteristics of Staphylococcus aureus causing bovine mastitis between 2014 and 2015. Front. Cell. Infect. Microbiol. $7: 127$.

Liu, H., S. Li, L. Meng, L. Dong, S. Zhao, X. Lan, J. Wang, and N. Zheng. 2017. Prevalence, antimicrobial susceptibility, and molecular characterization of Staphylococcus aureus isolated from dairy herds in northern China. J. Dairy Sci. 100:8796-8803.

Locatelli, C., P. Cremonesi, A. Caprioli, V. Carfora, A. Ianzano, A. Barberio, S. Morandi, A. Casula, B. Castiglioni, V. Bronzo, and P. Moroni. 2017. Occurrence of methicillin-resistant Staphylococcus aureus in dairy cattle herds, related swine farms, and humans in contact with herds. J. Dairy Sci. 100:608-619.

McCarthy, A. J., and J. A. Lindsay. 2010. Genetic variation in Staphylococcus aureus surface and immune evasion genes is lineage associated: Implications for vaccine design and host-pathogen interactions. BMC Microbiol. 10:173.

Mistry, H., P. Sharma, S. Mahato, R. Saravanan, P. A. Kumar, and V. Bhandari. 2016. Prevalence and characterization of oxacillin susceptible mecA-positive clinical isolates of Staphylococcus aureus causing bovine mastitis in India. PLoS One 11:e0162256.

Parisi, A., M. Caruso, G. Normanno, L. Latorre, R. Sottili, A. Miccolupo, R. Fraccalvieri, and G. Santagada. 2016. Prevalence, antimicrobial susceptibility and molecular typing of methicillin-resistant Staphylococcus aureus (MRSA) in bulk tank milk from southern Italy. Food Microbiol. 58:36-42.

Paterson, G. K., A. R. Larsen, A. Robb, G. E. Edwards, T. W. Pennycott, G. Foster, D. Mot, K. Hermans, K. Baert, S. J. Peacock, J. Parkhill, R. N. Zadoks, and M. A. Holmes. 2012. The newly described mecA homologue, mec $\mathrm{A}_{\mathrm{LGA} 251}$, is present in methicillinresistant Staphylococcus aureus isolates from a diverse range of host species. J. Antimicrob. Chemother. 67:2809-2813.

Peacock, S. J., C. E. Moore, A. Justice, M. Kantzanou, L. Story, K. Mackie, G. O'Neill, and N. P. Day. 2002. Virulent combinations of adhesin and toxin genes in natural populations of Staphylococcus aureus. Infect. Immun. 70:4987-4996.

Prenafeta, A., M. Sitjà, M. A. Holmes, and G. K. Paterson. 2014. Short communication: Biofilm production characterization of mecA and mecC methicillin-resistant Staphylococcus aureus isolated from bovine milk in Great Britain. J. Dairy Sci. 97:4838-4841.

Pu, W., Y. Su, J. Li, C. Li, Z. Yang, H. Deng, and C. Ni. 2014. High incidence of oxacillin-susceptible mecA-positive Staphylococcus aureus (OS-MRSA) associated with bovine mastitis in China. PLoS One 9:e88134.

Seixas, R., J. P. Santos, R. Bexiga, C. L. Vilela, and M. Oliveira. 2014. Short communication: Antimicrobial resistance and virulence characterization of methicillin-resistant staphylococci isolates from bovine mastitis cases in Portugal. J. Dairy Sci. 97:340-344.

Silva, N. C., F. F. Guimarães, M. P. Manzi, A. F. Júnior, E. GómezSanz, P. Gómez, H. Langoni, V. L. Rall, and C. Torres. 2014. Methicillin-resistant Staphylococcus aureus of lineage ST398 as cause of mastitis in cows. Lett. Appl. Microbiol. 59:665-669.
Song, J. W., S. J. Yang, S. Shin, K. S. Seo, Y. H. Park, and K. T. Park. 2016. Genotypic and phenotypic characterization of methicillin-resistant Staphylococcus aureus isolated from bovine mastitic milk in Korea. J. Food Prot. 79:1725-1732.

Tang, J., J. Chen, H. Li, P. Zeng, and J. Li. 2013. Characterization of adhesin genes, staphylococcal nuclease, hemolysis, and biofilm formation among Staphylococcus aureus strains isolated from different sources. Foodborne Pathog. Dis. 10:757-763.

Tung, H., B. Guss, U. Hellman, L. Persson, K. Rubin, and C. Rydén. 2000. A bone sialoprotein-binding protein from Staphylococcus aureus: A member of the staphylococcal Sdr family. Biochem. J. 345:611-619.

Türkyilmaz, S., S. Tekbiyik, E. Oryasin, and B. Bozdogan. 2010. Molecular epidemiology and antimicrobial resistance mechanisms of methicillin-resistant Staphylococcus aureus isolated from bovine milk. Zoonoses Public Health 57:197-203.

Vanderhaeghen, W., T. Cerpentier, C. Adriaensen, J. Vicca, K. Hermans, and P. Butaye. 2010. Methicillin-resistant Staphylococcus aureus (MRSA) ST398 associated with clinical and subclinical mastitis in Belgian cows. Vet. Microbiol. 144:166-171.

Wang, D., L. Zhang, X. Zhou, Y. He, C. Yong, M. Shen, O. Szenci, and B. Han. 2016. Antimicrobial susceptibility, virulence genes, and randomly amplified polymorphic DNA analysis of Staphylococcus aureus recovered from bovine mastitis in Ningxia, China. J. Dairy Sci. 99:9560-9569.

Wang, W., X. Lin, T. Jiang, Z. Peng, J. Xu, L. Yi, F. Li, S. Fanning, and Z. Baloch. 2018. Prevalence and characterization of Staphylococcus aureus cultured from raw milk taken from dairy cows with mastitis in Beijing, China. Front. Microbiol. 9:1123.

Xu, J., X. Tan, X. Zhang, X. Xia, and H. Sun. 2015. The diversities of staphylococcal species, virulence and antibiotic resistance genes in the subclinical mastitis milk from a single Chinese cow herd. Microb. Pathog. 88:29-38.

Yang, F., Q. Wang, X. Wang, L. Wang, X. Li, J. Luo, S. Zhang, and H. Li. 2016. Genetic characterization of antimicrobial resistance in Staphylococcus aureus isolated from bovine mastitis cases in northwest China. J. Integr. Agric. 15:2842-2847.

Yang, F., S. Zhang, X. Shang, L. Wang, H. Li, and X. Wang. 2018. Characteristics of quinolone-resistant Escherichia coli isolated from bovine mastitis in china. J. Dairy Sci. 101:6244-6252.

Yu, F., T. Li, X. Huang, J. Xie, Y. Xu, J. Tu, Z. Qin, C. Parsons, J. Wang, L. Hu, and L. Wang. 2012. Virulence gene profiling and molecular characterization of hospital-acquired Staphylococcus aureus isolates associated with bloodstream infection. Diagn. Microbiol. Infect. Dis. 74:363-368.

Zhang, L., J. Gao, H. W. Barkema, T. Ali, G. Liu, Y. Deng, S. Naushad, J. P. Kastelic, and B. Han. 2018. Virulence gene profiles: Alpha-hemolysin and clonal diversity in Staphylococcus aureus isolates from bovine clinical mastitis in china. BMC Vet. Res. 14:63.

\section{ORCIDS}

Feng Yang () https://orcid.org/0000-0003-0126-4246

Shidong Zhang @ https://orcid.org/0000-0002-3045-9010

Hang Zhang $\odot$ https://orcid.org/0000-0003-0127-5121 